\title{
Astronomical extinction over the ELT Moroccan sites from aerosol satellite data
}

\author{
El Arbi Siher ${ }^{1,2} \dagger$, Zouhair Benkhaldoun ${ }^{2}$ \\ and Aziza Bounhir ${ }^{2,3}$ \\ ${ }^{1}$ Faculté des Sciences et Techniques, Département de Physique, BP: 523, Béni Mellal, Maroc. \\ email: siher@ucam.ac.ma \\ ${ }^{2}$ Faculté des Sciences Semlalia, Département de Physique, LPHEA, Marrakech, Maroc. \\ email: zouhair@ucam.ac.ma \\ ${ }^{3}$ Faculté des Sciences et Techniques, Département de Physique Appliquée, Marrakech, Maroc. \\ email: bounhiraz@yahoo.fr
}

\begin{abstract}
Two Moroccan sites have been selected to be characterized for the ELT telescopes. Those sites are in the Atlas, between Oukaïmeden (where the national observatory is situated) and the Canary Islands. For this preliminary study, we have used the Nimbus-7 TOMS aerosol index to derive the astronomical extinction. This work builds on the findings of an earlier study which established the link between these two parameters over the Canary Islands.
\end{abstract}

Keywords. Site testing, atmospheric effects, telescopes

\section{Introduction}

In 1988, the astrophysics group of the University of Marrakech was funded for the construction of a national astronomical observatory. The first site chosen for this project was Oukaïmeden, located close to Marrakech in the High Atlas mountains at an altitude of $2700 \mathrm{~m}$. Several studies were made for the qualification of this site. Those studies allowed our group to develop experience in the field of site selection and site testing.

The geographical situation of the Atlas mountains makes them ideal candidate sites for the future Extremely Large Telescope (ELT). We therefore mobilized the members of our group to undertake qualitative studies of the selected sites. Several studies have started, namely, the measurement of the astronomical seeing, the observation of clouds and the influence of dust, coming from the south, on the state of the atmosphere. Our research focusses on two issues: (i) the characterization of the site from the point of view of the passage of the aerosols, and (ii) the determination the physical nature of these aerosols, an issue still under discussion (Siher, et al. (2002); Varela, et al. (2004)).

In an earlier paper (?), we showed how tropospheric aerosols can influence the astronomical extinction. We used the Nimbus-7 TOMS aerosol data and the observed astronomical extinction at the Observatorio del Roque de los Muchachos (ORM) on La Palma in the Canary Islands to establish this relationship.

In this work, we use the correlation found by Siher et al. (2002) to extract the astronomical extinction from the aerosol index over the period 1979-1992. Fortunately, all ELT Moroccan candidates sites are in the same TOMS/Nimbus7 pixel area. This method, not yet proved for sites other than ORM, gives a preliminary indication of the quality of these sites.

$\dagger$ Present address: Faculty of Sciences and Techniques, Department of Physics, BP: 523, Beni Mellal, Morocco. 


\section{Presentation of the ELT candidate sites}

A long list of a potential candidate sites for the ELT telescope was shortened in the framework of the ELT design study and the site working group proposed to focus on the study of four sites:

- Moroccan Anti-Atlas mountains: Adrar-n-Aklim $\left(2531 \mathrm{~m}, 30.1^{\circ} \mathrm{N}, 8.2^{\circ} \mathrm{W}\right)$ and Jebel Lekst $\left(2359 \mathrm{~m}, 29.7^{\circ} \mathrm{N}, 9^{\circ} \mathrm{W}\right)$;

- ORM, La Palma, Canary Islands;

- north of Paranal in Chile;

- The north western part of Argentina.

The Moroccan anti-Atlas mountains are close to the Atlantic Ocean and are influenced by the wind coming from the west.

\section{Preliminary determination of astronomical extinction from aerosol index over Moroccan ELT sites}

The correlation between aerosol index (AI) and astronomical extinction (AE) as found by Siher et al. (2002) is given by:

$$
A E=A I * 0.16+0.14
$$

This equation obtained for the ORM observatory is still under verification for other sites.

Figure 1 shows the evolution of the astronomical extinction for the proposed ELT sites, derived from the aerosol index using equation 3.1 for the period 1979-1992. The Nimbus-7 TOMS aerosol indices (AI) are greater than about 0.7 (absorbing aerosol), which corresponds to a threshold of about $0.2 \mathrm{mag} /$ airmass for the derived AE values.

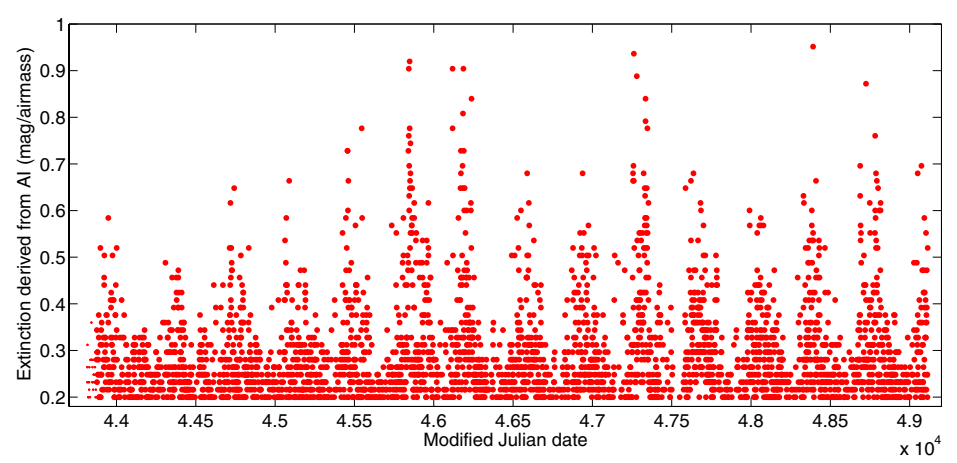

Figure 1. Evolution of the derived extinction from the aerosol index over the ELT sites

Figure 3 is a histogram showing the distribution of extinction values derived from the aerosol index. In this figure, we note the presence of a one-year period showing the seasonal variation in extinction. The high values occur in April-May-June and the minima occur in the winter. By contrast, in the High-Atlas mountains (Oukaïmeden), the high values occur in the summer (June-July and August) (Siher, et al. (2002). Figure 2 clearly shows this seasonal effect by presenting the monthly variation, modulo one year, of the astronomical extinction derived from the aerosol index over the ELT sites. This figure shows that the predominant intervals are less than $0.25 \mathrm{mag} /$ airmass; therefore, we can say that this location is not strongly influenced by the Saharan dust. 


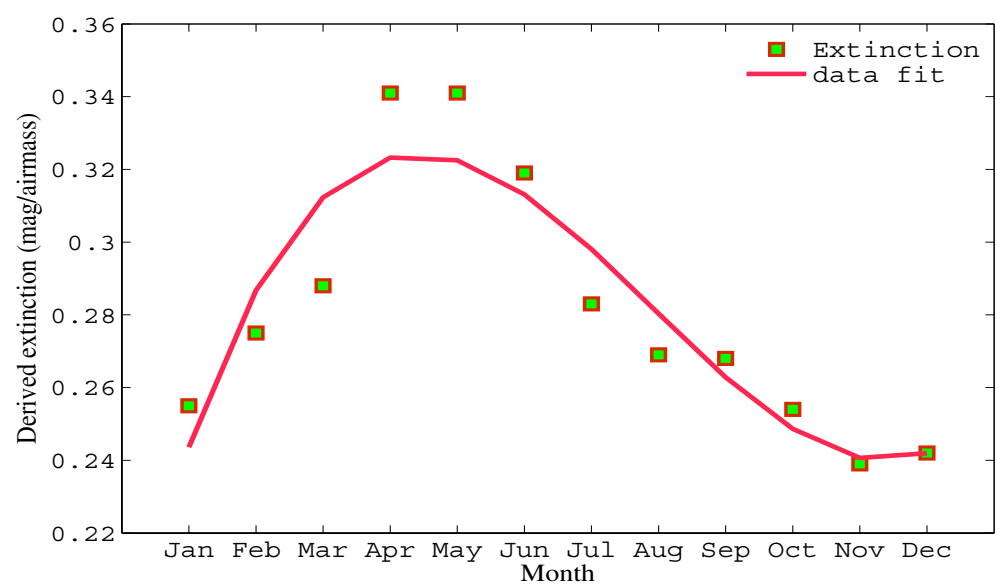

Figure 2. Monthly variation, modulo one year, of the derived extinction at the ELT sites. The continuous line is a fit showing a clear seasonal variation.

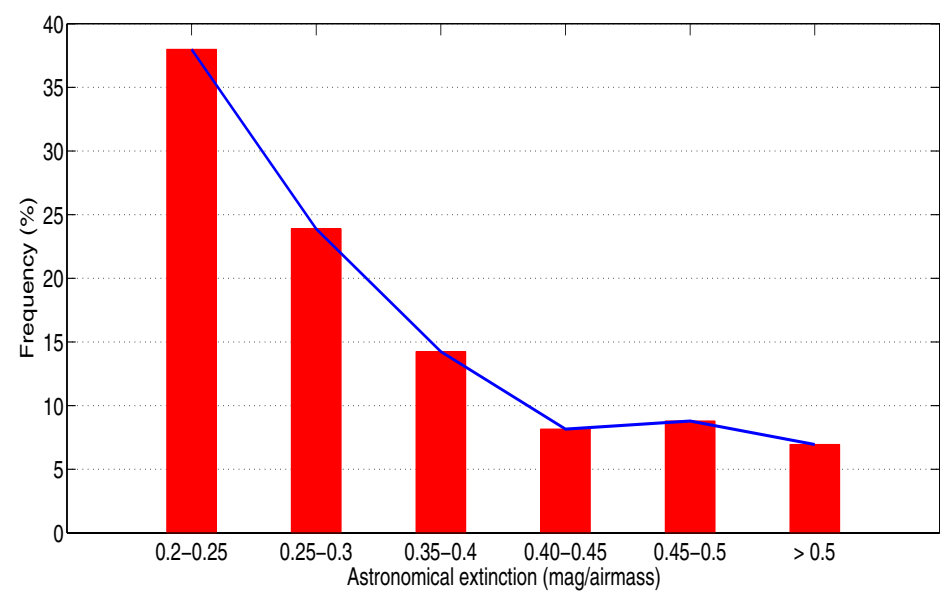

Figure 3. Percent of predominating intervals of extinction

Table 1. Astronomical extinction over Moroccan ELT sites and ORM. Values are derived from aerosol index.

\begin{tabular}{lcccccccccccccc}
\hline Year & 79 & 80 & 81 & 82 & 83 & 84 & 85 & 86 & 87 & 88 & 89 & 90 & 91 & 92 \\
\hline ORM & .38 & .39 & .40 & .36 & .37 & .39 & .41 & .39 & .36 & .43 & .39 & .38 & .35 & .38 \\
Moroccan ELT sites & .27 & .26 & .27 & .27 & .27 & .31 & .30 & .27 & .29 & .31 & .29 & .28 & .28 & .28 \\
\hline
\end{tabular}

To compare our sites to the ORM observatory, we present in Table 1 the yearly means of the extinction derived from aerosol index over both ELT sites and the ORM site. As a caveat, we caution that ORM values represent only summer period means, but, for the Moroccan ELT sites, values are calculated over the entire year. This difference results in a higher derived annual mean extinction over the ORM observatory compared to the Moroccan sites. 


\section{Conclusions}

This study uses the correlation between aerosol index and astronomical extinction established over the ORM observatory to derive the extinction over the Moroccan ELT sites. We find that this derived extinction exhibits a seasonal variation and that the extinction is predominantly less than $0.25 \mathrm{mag} /$ airmass. We further conclude that the Moroccan ELT sites are not strongly influenced by the Saharan dust. This study confirms that these Moroccan sites are good potential sites for the ELT.

\section{Acknowledgements}

We thank the European Southern Observatory, especially Marc Sarazin, for the financing our participation in the General Assembly of the IAU in Prague, the IAU Secretary General for paying the registration fee and the Cadi Ayyad University for the financing of the airfare. Our thanks also to NASA for the use of the Nimbus-7 TOMS data.

\section{References}

Siher, E.A., Ortolani, S., Sarazin, M., Benkhaldoun, Z. 2002, SPIE 5489, 138

Siher, E.A., Benkhaldoun, Z., Fossat, E. 2002, Experimental Astronomy 13, 159

Varela, Antonia M., Fuensalida, Jesús J., Muñoz-Tuñón, C., Rodríguez, E., José, M., GarcíaLorenzo, B., Cuevas, E. 2004, SPIE 5489, 105 